\title{
The Total Number of Microorganisms Grown on Wheat Obtained in South-East of Albania
}

\author{
Lorena Memushaj ${ }^{1}$, Donika Prifti \\ ${ }^{1,2}$ Department of Chemistry, Faculty of Natural Sciences, University of Tirana, Albania
}

\begin{abstract}
The purpose of this study is to create a general overview on microbiological characteristics determine on the surface and epiderm of the wheat. The wheat samples were obtained in the south-east regions of Albania during March-June period, 2015. Microbiological evaluation was done in 4 different media for total bacteria, yeasts and mold. The total number of microorganisms grown on the surface of wheat was determined by the method of placing the suspensions on solid medium. After incubation at $26^{\circ} \mathrm{C}$ (for mold,) and at $30{ }^{\circ} \mathrm{C}$ (for bacteria), the colonies were counted. The concentration of microorganisms in the original samples was calculated from plates with 25 - 250 colonies. The calculation of total number of microorganisms was done by assuming that each colony grew from a single cell. A very important point of this study is the identification of mold based on colony growth, cultural and phenotypic characteristics. Mould species present in wheat were determined by direct microscopic observation. By identification of moulds and classification in species and genus there was noticed mainly Ascomycete classes especially Aspergillus spp. In general wheat samples which have been analyzed were within standards.
\end{abstract}

Keywords: wheat, mold, surface, Aspergillus spp.

\section{Introduction}

Wheat is actually a grass and belongs to the genus Triticum species aestivum. Wheat is grown globally and is the second most important cereal crop in the world behind maize and ahead of rice. Wheat (Triticum spp.) is a cereal grain, originally from the Levant region of the Near East but now cultivated worldwide. In 2013, world production of wheat was 713 million tons, making it the third most produced cereal after maize (1,016 million tons) and rice (745 million tons).

Wheat was the second most-produced cereal in 2009; world production in that year was 682 million tons, after maize (817 million tons), and with rice as a close third (679 million tons). Wheat is probably the most common cereal available all over the world and is in even higher demand in recent years due to its abundant health benefits. Over the years, wheat has shown itself to be one of the most successful and sustainable cereals crops in the world. Microorganisms that contaminate cereal grains may come from air, dust, soil, water, insects, rodents, birds, animals, humans, storage and shipping containers, and handling and processing equipment. Many factors that are a part of the environment influence microbial contamination of cereals, including rainfall, drought, humidity, temperature, sunlight, frost, soil conditions, wind, insect, bird and rodent activity, harvesting equipment, use of chemicals in production versus organic production, storage and handling, and moisture control. The microflora of cereals and cereal products is varied and includes mold, yeast and bacteria[12].

\section{Materials and methods}

The purpose of this study is to create a general overview on microbiological characteristics determine on the surface and epiderm of the wheat. Five samples were obtained in southeast regions of Albania during March-June period, 2015. Experimental work has been developed in the laboratory of microbiology at the Faculty of Natural Sciences of Tirana, Albania. This study was based on fungus present in wheat's germ. Total numbers of fungi was determined by dilution techniques [8]. The samples were obtained in strictly sterile conditions in order to avoid the impact of environmental and air microorganisms. They were taken in different parts of silos for a better presentation. This was done because microorganisms are different in the upper and inner layer of silos [7].

The total numbers of molds were determined by the method of placing the suspensions on solid medium (Czapek dox agar, Potato doxtrose agar, Malt extract agar, Plate count agar). It was prepared three dilutions with 2 parallels. After incubation at $26^{\circ} \mathrm{C}$ the colonies were counted. The calculation of total number of microorganisms was done by assuming that each colony grew from a single cell [9]. $10 \mathrm{~g}$ wheat was taken and thrown into an Erlenmeyer with $90 \mathrm{ml}$ of sterile water. There were used three dilutions and it was cultivated 2 parallels with $1 \mathrm{ml}$ suspension in each plate [11].

Very important point of this study was the identification of molds that were grown in all media. The identification of mold was based on colony growth, cultural and phenotypic characteristics. Some of them were determined by direct microscopic observation. They were classified in 5 species: Rhizopus spp. and Mucor spp. [8], Aspergillus spp. [6], Penicillium spp. [10], Fungi imperfecti spp. [1]

\section{Results and Discussion}

The result for total number of microorganisms, (bacteria, yeast and mold) was shown in Table 1 for five samples. The calculation was done for four solid medium (Czapek dox agar, Potato doxtrose agar, Malt extract agar, Plate count agar). The results were observed that the total number of bacteria grown on the surface were some times higher than the epiderm. There was one exception for sample 3 , wherein the total number of bacteria was some times higher in

\section{Volume 4 Issue 11, November 2015}




\section{International Journal of Science and Research (IJSR) \\ ISSN (Online): 2319-7064 \\ Index Copernicus Value (2013): 6.14 | Impact Factor (2014): 5.611}

epiderm than the surface. There was mainly Pseudomonas herbicola species which are characteristics for young wheat. The total number of yeast were little higher on the surface than the epiderm. The total number of mold was two times higher on the surface than the epiderm.

A very important point of this study was the identification of mold which was grown in all media. The identification of mold was based on colony growth, cultural and phenotypic characteristics. Some of them were determined by direct microscopic observation.

Table 1: Total number of microorganisms

\begin{tabular}{|c|c|c|c|c|c|c|c|c|c|c|c|c|c|c|c|}
\hline \multicolumn{16}{|c|}{ Sample 1} \\
\hline \multirow{3}{*}{ 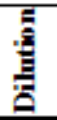 } & \multirow{3}{*}{ 롤 } & \multicolumn{7}{|c|}{ Microorganisms on the surface } & \multicolumn{7}{|c|}{ Microorganisms on the ep iderm } \\
\hline & & \multicolumn{2}{|c|}{ Bacteria } & Yeast & \multicolumn{4}{|c|}{ Mold } & \multicolumn{2}{|c|}{ Bacteria } & Yeast & \multicolumn{4}{|c|}{ Mold } \\
\hline & & PCA & PDA & MA & PCA & PDA & MA & Czapek & PCA & PDA & MA & PCA & PDA & MA & Czapek \\
\hline \multirow{4}{*}{$10^{-1}$} & 1 & Too many & Too many & 6 & 1 & 14 & 4 & 4 & 100 & Too many & 9 & 1 & 3 & 2 & 2 \\
\hline & 2 & Too many & Too many & 12 & 0 & 10 & 0 & 5 & 98 & Too many & 0 & 0 & 3 & 1 & 2 \\
\hline & Average & Toomany & Too many & 9 & 0.5 & 12 & 2 & 4.5 & 99 & Toomany & 4.5 & 0.5 & 3 & 1.5 & 2 \\
\hline & T otal & \multicolumn{2}{|c|}{ T oo many } & 90 & \multicolumn{4}{|c|}{190} & \multicolumn{2}{|c|}{990} & 45 & \multicolumn{4}{|c|}{70} \\
\hline \multirow{4}{*}{$10^{-2}$} & 1 & Too many & Too many & 7 & 1 & 1 & 0 & 3 & 16 & Too many & 4 & 0 & 0 & 0 & 1 \\
\hline & 2 & Too many & Too many & 5 & 1 & 0 & 0 & 1 & 13 & Too many & 2 & 2 & 0 & 0 & 0 \\
\hline & Average & Too many & Too many & 6 & 1 & 0.5 & 0 & 2 & 14.5 & Too many & 3 & 1 & 0 & 0 & 0.5 \\
\hline & Total & \multicolumn{2}{|c|}{ p.n. } & 600 & \multicolumn{4}{|c|}{350} & \multicolumn{2}{|c|}{1450} & 300 & \multicolumn{4}{|c|}{150} \\
\hline \multirow{4}{*}{$10^{-3}$} & 1 & 74 & 25 & 5 & 0 & 0 & 0 & 2 & 0 & 23 & 1 & 0 & 0 & 0 & 1 \\
\hline & 2 & 30 & 32 & 5 & 1 & 0 & 0 & 1 & 0 & 25 & 5 & 0 & 0 & 0 & 0 \\
\hline & Average & 52 & 28.5 & 5 & 0.5 & 0 & 0 & 1.5 & 0 & 24 & 3 & 0 & 0 & 0 & 0.5 \\
\hline & T otal & \multicolumn{2}{|c|}{80500} & 5000 & \multicolumn{4}{|c|}{2000} & \multicolumn{2}{|c|}{24000} & 3000 & \multicolumn{4}{|c|}{500} \\
\hline \multicolumn{16}{|c|}{ Sample 2} \\
\hline & $=$ & & Microor: & anism & on th & surf: & & & & Microo & ganism & on $t$ & der & & \\
\hline 音 & ह & Bact & teria & Yeast & & & old & & & eria & Yeast & & & ld & \\
\hline 豆 & 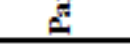 & PCA & PDA & MA & $\mathrm{PCA}$ & PDA & MA & Czapek & PCA & PDA & MA & PCA & PDA & $\mathrm{MA}$ & Czapek \\
\hline & 1 & Too many & Too many & 18 & 0 & 17 & 4 & 16 & 160 & Toomany & 18 & 1 & 6 & 0 & 3 \\
\hline $10^{-1}$ & 2 & Too many & Too many & 16 & 0 & 0 & 0 & 12 & 154 & Toomany & 9 & 0 & 5 & 0 & 1 \\
\hline $10^{-2}$ & Average & Toomany & Too many & 17 & 0 & 8.5 & 2 & 14 & 157 & Toomany & 13.5 & 0.5 & 5.5 & 0 & 2 \\
\hline & T otal & $\mathrm{T} 001$ & many & 170 & & & 45 & & & & 135 & & & 0 & \\
\hline & 1 & Too many & Too many & 9 & 0 & 8 & 1 & 0 & 100 & Toomany & 6 & 0 & 2 & 0 & 2 \\
\hline & 2 & Too many & Too many & 7 & 0 & 5 & 0 & 0 & 98 & Toomany & 6 & 0 & 1 & 0 & 0 \\
\hline $10^{\circ}$ & Average & Too many & Too many & 8 & 0 & 6.5 & 0.5 & 0 & 99 & Too many & 6 & 0 & 1.5 & 0 & 1 \\
\hline & Total & Too 1 & many & 800 & & & 00 & & & & 600 & & & 0 & \\
\hline & 1 & 90 & 34 & 3 & 0 & 3 & 0 & 1 & 60 & 22 & 2 & 0 & 2 & 0 & 0 \\
\hline & 2 & 80 & 29 & 5 & 0 & 0 & 0 & 0 & 51 & 30 & 2 & 0 & 1 & 0 & 0 \\
\hline $10^{2}$ & Average & 85 & 31.5 & 4 & 0 & 1.5 & 0 & 0.5 & 55.5 & 26 & 2 & 0 & 1.5 & 0 & 0 \\
\hline & T otal & 116 & 500 & 4000 & & & 00 & & & & 2000 & & & 00 & \\
\hline
\end{tabular}

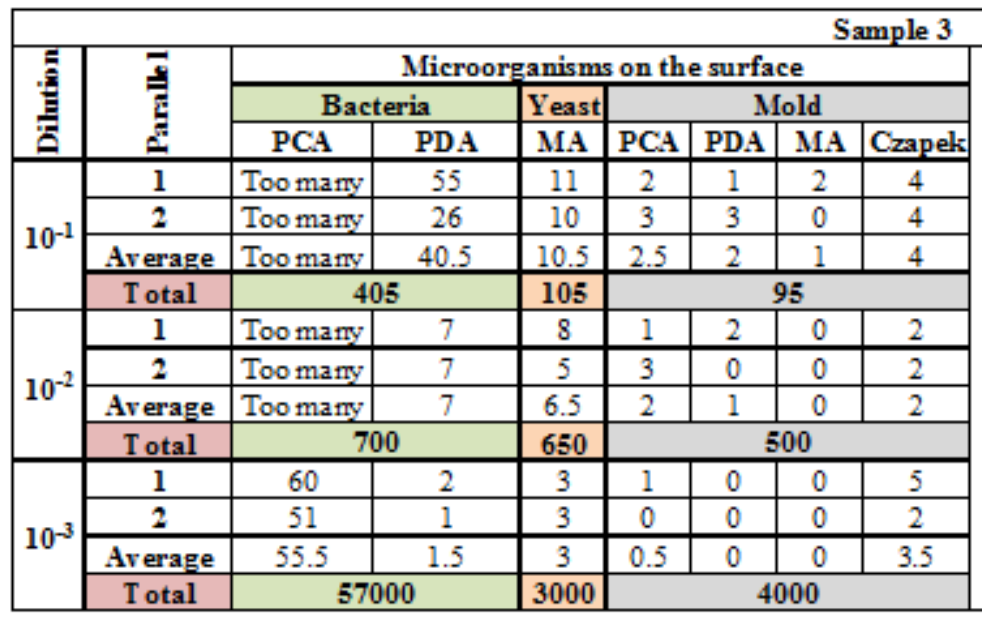

\begin{tabular}{|c|c|c|c|c|c|c|}
\hline \multicolumn{7}{|c|}{ Microorganisms on the ep iderm } \\
\hline \multicolumn{2}{|c|}{ Bacteria } & \multirow{2}{*}{\begin{tabular}{|c|} 
Yeast \\
MA \\
\end{tabular}} & \multicolumn{4}{|c|}{ Mold } \\
\hline PCA & PDA & & $\mathrm{PCA}$ & PDA & MA & Czapel \\
\hline Too many & 180 & 9 & 2 & 2 & 1 & 3 \\
\hline Too many & 182 & 5 & 1 & 0 & 0 & 1 \\
\hline Toomany & 181 & 7 & 1.5 & 1 & 0.5 & 2 \\
\hline \multicolumn{2}{|c|}{1810} & 70 & \multicolumn{4}{|c|}{50} \\
\hline Too many & 37 & 8 & 3 & 0 & 0 & 2 \\
\hline Too many & 13 & 3 & 0 & 0 & 0 & 1 \\
\hline Too many & 25 & 5.5 & 1.5 & 0 & 0 & 1.5 \\
\hline \multicolumn{2}{|c|}{2500} & 550 & \multicolumn{4}{|c|}{300} \\
\hline 41 & 1 & 5 & 0 & 0 & 0 & 1 \\
\hline 38 & 1 & 0 & 0 & 0 & 0 & 0 \\
\hline 39.5 & 1 & 2.5 & 0 & 0 & 0 & 0.5 \\
\hline \multicolumn{2}{|c|}{40500} & 2500 & \multicolumn{4}{|c|}{500} \\
\hline
\end{tabular}




\section{International Journal of Science and Research (IJSR) \\ ISSN (Online): 2319-7064}

Index Copernicus Value (2013): 6.14 | Impact Factor (2014): 5.611

\begin{tabular}{|c|c|c|c|c|c|c|c|c|c|c|c|c|c|c|c|}
\hline \multicolumn{16}{|c|}{ Sample 4} \\
\hline \multirow{3}{*}{ 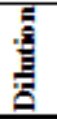 } & \multirow{3}{*}{ ت气 } & \multicolumn{7}{|c|}{ Microorganisms on the surface } & \multicolumn{7}{|c|}{ Microorganisms on the ep iderm } \\
\hline & & \multicolumn{2}{|c|}{ Bacteria } & Yeast & \multicolumn{4}{|c|}{ Mold } & \multicolumn{2}{|c|}{ Bacteria } & \multirow{2}{*}{\begin{tabular}{c|} 
Yeast \\
MA
\end{tabular}} & \multicolumn{4}{|c|}{ Mold } \\
\hline & & PCA & PDA & MA & PCA & PDA & MA & Czapek & PCA & PDA & & $\mathrm{PCA}$ & PDA & MA & Czapek \\
\hline \multirow{4}{*}{$10^{-1}$} & 1 & Too many & Too many & 10 & 4 & 2 & 1 & 1 & 56 & 0 & 4 & 1 & 1 & 0 & 1 \\
\hline & 2 & Too many & Too many & 2 & 0 & 1 & 0 & 0 & 63 & 0 & 0 & 3 & 0 & 0 & 2 \\
\hline & Average & Toomany & Too many & 6 & 2 & 1.5 & 0.5 & 0.5 & 59.5 & 0 & 2 & 2 & 0.5 & 0 & 1.5 \\
\hline & T otal & \multicolumn{2}{|c|}{ T oo many } & 60 & \multicolumn{4}{|c|}{45} & \multicolumn{2}{|c|}{595} & 20 & \multicolumn{4}{|c|}{40} \\
\hline \multirow{4}{*}{$10^{-2}$} & 1 & 173 & 0 & 5 & 0 & 1 & 0 & 1 & 9 & 0 & 5 & 0 & 1 & 0 & 0 \\
\hline & 2 & 179 & 0 & 5 & 1 & 0 & 0 & 0 & 9 & 0 & 0 & 0 & 0 & 0 & 0 \\
\hline & Average & 176 & 0 & 5 & 0.5 & 0.5 & 0 & 0.5 & 9 & 0 & 2.5 & 0 & 0.5 & 0 & 0 \\
\hline & T otal & \multicolumn{2}{|c|}{17600} & 500 & \multicolumn{4}{|c|}{150} & \multicolumn{2}{|c|}{900} & 250 & \multicolumn{4}{|c|}{50} \\
\hline \multirow{4}{*}{$10^{-3}$} & 1 & 18 & 0 & 2 & 1 & 0 & 0 & 3 & 3 & 0 & 1 & 0 & 0 & 0 & 1 \\
\hline & 2 & 16 & 0 & 3 & 0 & 0 & 0 & 1 & 4 & 0 & 0 & 0 & 0 & 0 & 0 \\
\hline & Average & 17 & 0 & 2.5 & 0.5 & 0 & 0 & 2 & 3.5 & 0 & 0.5 & 0 & 0 & 0 & 0.5 \\
\hline & T otal & \multicolumn{2}{|c|}{17000} & 2500 & \multicolumn{4}{|c|}{2500} & \multicolumn{2}{|c|}{3500} & 500 & \multicolumn{4}{|c|}{500} \\
\hline & & & & & & & & imple 5 & & & & & & & \\
\hline $\bar{E}$ & $=$ & & Microor & anism & on th & surfs & & & & Miero & ganisms & on the & epider & & \\
\hline 車 & ह & Bac & teria & Yeast & & & old & & & & Yeast & & & ld & \\
\hline & $\approx$ & PCA & PDA & MA & PCA & PDA & MA & Czapek & PCA & PDA & MA & PCA & PDA & MA & Czapek \\
\hline & 1 & 106 & Too many & 6 & 0 & 9 & 0 & 1 & 38 & 9 & 1 & 0 & 0 & 0 & 0 \\
\hline 10-1 & 2 & 80 & Too many & 5 & 1 & 10 & 0 & 1 & 36 & 8 & 3 & 0 & 2 & 0 & 1 \\
\hline $10^{-2}$ & Average & 93 & Too many & 5.5 & 0.5 & 9.5 & 0 & 1 & 37 & 8.5 & 2 & 0 & 1 & 0 & 0.5 \\
\hline & T otal & & 30 & 55 & & & 10 & & & & 20 & & & 5 & \\
\hline & 1 & 5 & 85 & 2 & 1 & 1 & 0 & 0 & 4 & 2 & 2 & 0 & 0 & 0 & 0 \\
\hline $10^{-2}$ & 2 & 3 & 74 & 8 & 0 & 2 & 0 & 0 & 2 & 0 & 1 & 0 & 0 & 0 & 0 \\
\hline $10^{-2}$ & Average & 4 & 79.5 & 5 & 0.5 & 1.5 & 0 & 0 & 3 & 1 & 1.5 & 0 & 0 & 0 & 0 \\
\hline & T otal & & 50 & 500 & & & 00 & & & & 150 & & & 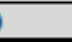 & \\
\hline & 1 & 0 & 5 & 0 & 0 & 0 & 0 & 0 & 1 & 0 & 0 & 0 & 0 & 0 & 0 \\
\hline $10^{-3}$ & 2 & 0 & 1 & 0 & 0 & 0 & 0 & 0 & 0 & 0 & 0 & 0 & 0 & 0 & 0 \\
\hline $10^{\circ}$ & Average & 0 & 3 & 0 & 0 & 0 & 0 & 0 & 0.5 & 0 & 0 & 0 & 0 & 0 & 0 \\
\hline & T otal & & 00 & 0 & & & $\underline{0}$ & & & & 0 & & & & \\
\hline
\end{tabular}

By identification of mold and classification in species and genius there was noticed mainly Ascomycete class especially Aspergillus and Penicillium species. Aspergillus spp. especially A.candidus. Their value in percentage was from $23.1-100 \%$ on the surface and $0-75 \%$ on the epiderm. This shows that wheat used in industrial mill companies are not young, but are stored wheat for a while. The value of Fungi imperfecti class was from $0-63.3 \%$ on the surface and 0 $33.3 \%$ on the epiderm. In general wheat samples which have been analyzed were within standards. The results are presented in Table 2, 3, Figure 1, 2. The sample with the highest number of mold, especially Aspergillus spp. was sample 2 .

This shows that wheat grain is a very suitable media for growing of microorganisms especially mold. Mill companies have to be careful during wheat preparation because mold grown would be cause a potencial risk.

Table 2: The identification and classification of mold grown on the surface of the wheat.

\begin{tabular}{|c|c|c|c|c|c|c|c|c|c|}
\hline Class & \multicolumn{3}{|c|}{ Phycomycetes } & \multicolumn{4}{|c|}{ Ascomycetes } & \multicolumn{2}{|l|}{ Fungi imperfecti } \\
\hline Genus & Rhizopus & Mucor & & Aspergillus & & Penicillium & & & \\
\hline Sample 1 & & & & $\begin{array}{l}\text { A.candidus } \\
\text { A.flavus } \\
\text { A.terreus }\end{array}$ & $\begin{array}{c}11 \\
1 \\
1\end{array}$ & Penicillium spp. & 5 & $\begin{array}{l}\text { Fungi imperfecti } \\
\text { Helminthosporium spp. } \\
\text { Cladosporium spp. }\end{array}$ & $\begin{array}{c}10 \\
19 \\
2\end{array}$ \\
\hline Nr. Total & $\mathbf{0}$ & & $\mathbf{0}$ & & 13 & & 5 & & 31 \\
\hline Sample 2 & & & & \begin{tabular}{|l} 
A.candidus \\
A.flavus \\
A.terreus \\
A.versicolor \\
A.glaucus
\end{tabular} & $\begin{array}{c}32 \\
3 \\
2 \\
1 \\
4 \\
\end{array}$ & $\begin{array}{l}\text { Penicillium spp. } \\
\text { P.islandicum }\end{array}$ & $\begin{array}{c}18 \\
1\end{array}$ & $\begin{array}{l}\text { Trichothecium roseum } \\
\text { Helminthosporium spp. } \\
\text { Cladosporium spp. }\end{array}$ & $\begin{array}{l}3 \\
2 \\
1\end{array}$ \\
\hline Nr. Total & $\mathbf{0}$ & & $\mathbf{0}$ & & 42 & & 19 & & 6 \\
\hline Sample 3 & & Mucor & 1 & \begin{tabular}{|l} 
A.candidus \\
A.terreus
\end{tabular} & $\begin{array}{l}8 \\
1\end{array}$ & & & & \\
\hline Nr. Total & $\mathbf{0}$ & & 1 & & 9 & & $\mathbf{0}$ & & $\mathbf{0}$ \\
\hline Sample 4 & & & & $\begin{array}{l}\text { A.candidus } \\
\text { A.niger }\end{array}$ & $\begin{array}{l}3 \\
2 \\
\end{array}$ & & & & \\
\hline Nr. Total & 0 & & $\mathbf{0}$ & & 5 & & $\mathbf{0}$ & & $\mathbf{0}$ \\
\hline Sample 5 & & Mисог & 2 & $\begin{array}{l}\text { A.candidus } \\
\text { A.flavus }\end{array}$ & $\begin{array}{l}4 \\
2\end{array}$ & $\begin{array}{l}\text { Penicillium spp. } \\
\text { P.islandicum } \\
\text { P.cyclopium }\end{array}$ & $\begin{array}{c}14 \\
1 \\
1\end{array}$ & Helminthosporium spp. & 2 \\
\hline Nr. Total & $\mathbf{0}$ & & 2 & & 6 & & 16 & & 2 \\
\hline
\end{tabular}

Volume 4 Issue 11, November 2015 


\section{International Journal of Science and Research (IJSR) \\ ISSN (Online): 2319-7064 \\ Index Copernicus Value (2013): 6.14 | Impact Factor (2014): 5.611}

Table 3: The identification and classification of mold grown on the epiderm of the wheat.

\begin{tabular}{|c|c|c|c|c|c|c|}
\hline Class & \multicolumn{2}{|c|}{ Phycomycetes } & \multicolumn{2}{|c|}{ Ascomycetes } & \multicolumn{2}{|l|}{ Fungi imperfecti } \\
\hline Genus & Rhizopus & Mucor & Aspergillus & Penicillium & & \\
\hline Sample 1 & & & \begin{tabular}{|ll} 
A.candidus & 2 \\
A.flavus & 1 \\
A.terreus & 2
\end{tabular} & Penicillium spp. 6 & $\begin{array}{l}\text { Trichothecium roseum } \\
\text { Helminthosporium spp. }\end{array}$ & \\
\hline Nr. Total & $\mathbf{0}$ & $\mathbf{0}$ & 5 & 6 & & 2 \\
\hline Sample 2 & & & 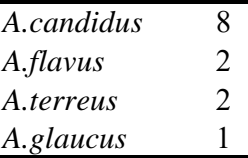 & Penicillium spp. $\quad 10$ & Trichothecium roseum & 1 \\
\hline Nr. Total & $\overline{0}$ & $\overline{0}$ & 13 & 10 & & 1 \\
\hline Sample 3 & & & \begin{tabular}{|ll} 
A.candidus & 1 \\
A.terreus & 2 \\
\end{tabular} & & Helminthosporium spp. & 1 \\
\hline Nr. Total & $\mathbf{0}$ & $\mathbf{0}$ & 3 & $\mathbf{0}$ & & 1 \\
\hline Sample 4 & R.nigricans & & & & & \\
\hline Nr. Total & 1 & $\mathbf{0}$ & $\mathbf{0}$ & $\mathbf{0}$ & & $\mathbf{0}$ \\
\hline Sample 5 & & & A.niger & Penicillium spp. 1 & Cladosporium spp. & 1 \\
\hline Nr. Total & $\mathbf{0}$ & $\mathbf{0}$ & 1 & 1 & & 1 \\
\hline
\end{tabular}

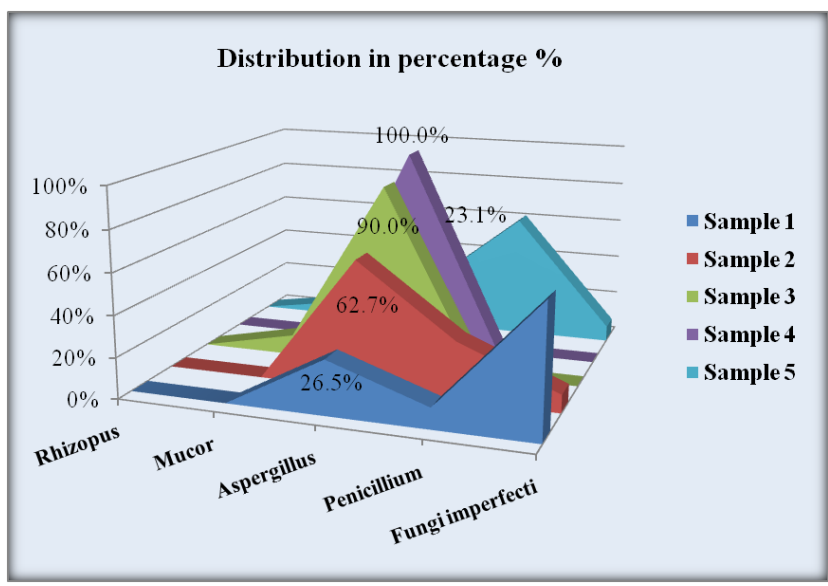

Figure 1: Distribution, in percentage \%, of mold grown on the surface of the wheat.

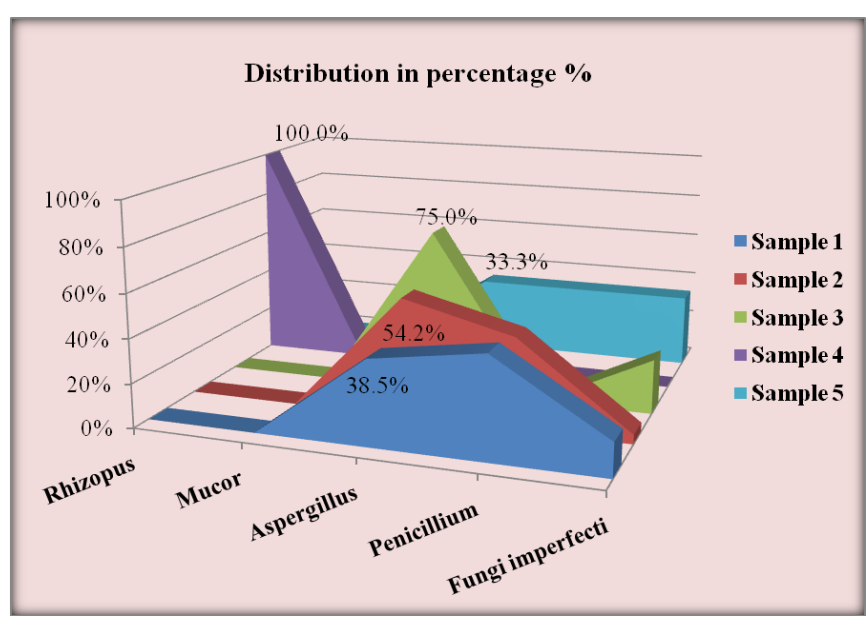

Figure 2: Distribution, in percentage \%, of mold grown on the epiderm of the wheat.

\section{Conclusion}

Because of their extensive use as human foods and livestock feeds, the microbiology and safety of cereal grains and cereal products is a very important area. The sources of microbial contamination of cereals are many, but all are traceable to the environment in which grains are grown, handled, and processed. Microorganisms that contaminate cereal grains may come from air, dust, soil, water, insects, rodents, birds, animals, humans, storage and shipping containers, and handling and processing equipment. From all the experimental work, we may recommend that the industrial mill companies should pay attention to preparation for milling processes, such as: cleaning, sorting, conditioning of the wheat because wheat is the basic food in our life.

\section{References}

[1] Barnet, H.L.; B. Hunter(1972), Ilustrated Genera of Imperfect Fungi. 3rd ed., Burgess Publishing Company, California.

[2] Berghofer, L.K., Hocking, A.D., Miskelly, D., Jansson, E., (2003). Microbiology of wheat and flour milling in Australia. Int. J. Food Microbial. 85, 137-149.

[3] Guarro, J., and J. Gene. (1992), Fusarium infections. Criteria for the identification of the responsible species. Mycoses 35, 109-114.

[4] Hocking, A.D. (2003).Microbiological facts and fictions in grain storage, Food Science Australia, PO Box 52, North Ryde, NSW 1670, 55 -58.

[5] ICMSF (1998), Microorganisms in Foods: 6 Microbial Ecology of Food Commodities. Blackie Academic and Professional, London, 313-346.

[6] J.A.Von ARX( 1970), The genera of fungi sporulation in pure culture-LEHRE-J.Cramer. Pelcyar, M.J. (1986), Microbiologjia, ZANICHELLI, Bologna, 724738.

[7] King, A.D., Hocking, A.D., Pitt, J.I., (1979), Dichloran-rose bengal medium for enumeration and isolation of molds from foods. Appl. Environ Microbial. 37, 959-964.

[8] Pitt, J.I.; Hocking, A.D.(2009) Fungi and Food Spoilage, 3rd ed.; Springer: Dordrecht, The Netherlands. 


\section{International Journal of Science and Research (IJSR) \\ ISSN (Online): 2319-7064}

Index Copernicus Value (2013): 6.14 | Impact Factor (2014): 5.611

[9] Prifti D. (1980): Wheat's microrganisms and their impact on the physic-chemical and technological changes during storage, Tirane, Albania.

[10] Raper, K.B.; Thom, C. A (1949), Manual of Penicillia; The Wil liams \& Wilkins Company: Baltimore, MD, USA.

[11] Voshtina, P.; Prifti, D (2007). Food Microbilogy, Tirana.

[12] www.organicfacts.net 